MITSUBISHI ELECTRIC RESEARCH LABORATORIES

http://www.merl.com

\title{
Adaptive Antenna Selection at Mobile Stations for SDMA in WiMAX Networks
}

\author{
Tairan Wang, Zhifeng Tao, Andreas Molisch, Philip Orlik, Jinyun Zhang
}

TR2009-038 September 03, 2009

\begin{abstract}
The IEEE 802.16/WiMAX standards has fully embraced multi-antenna technology and can, thus, deliver robust performance and high transmission rates. Nevertheless, due to its inherent cost concerns, a WiMAX mobile station (MS) should preferably contain fewer radio frequency $(\mathrm{RF})$ chains than antenna elements. Thus, antenna selection, wherein a subset of antennas is dynamically selected to connect to the limited number of RF chains for transceiving, is a highly appealing performance enhancement technique for multiantenna WiMAX terminals. In this paper, a novel protocol for antenna selection in space division multiple access (SDMA) transmission is developed for the next-generation IEEE 802.16 mobile stations. Both locally and globally optimal selection rules are considered at the base station (BS). The proposed protocol can readily accommodate various channel situations (e.g., reciprocal and non-reciprocal channels). As demonstrated by analysis and simulation, the proposed protocol delivers considerable performance improvement over conventional IEEE 802.16 terminals that lack antenna selection capability. Moreover, the proposed protocol leverages the existing signaling method defined in IEEE 802.16, thereby incurring a negligible signaling overhead and requiring only minimal modifications of the standard.
\end{abstract}

International Wireless Communications and Mobile Computing Conf.

This work may not be copied or reproduced in whole or in part for any commercial purpose. Permission to copy in whole or in part without payment of fee is granted for nonprofit educational and research purposes provided that all such whole or partial copies include the following: a notice that such copying is by permission of Mitsubishi Electric Research Laboratories, Inc.; an acknowledgment of the authors and individual contributions to the work; and all applicable portions of the copyright notice. Copying, reproduction, or republishing for any other purpose shall require a license with payment of fee to Mitsubishi Electric Research Laboratories, Inc. All rights reserved. 



\section{Adaptive Antenna Selection at Mobile Stations for SDMA in WiMAX Networks}

\author{
Tairan Wang \\ Department of Electrical and \\ Computer Engineering \\ University of Minnesota \\ Minneapolis, MN 55455, USA \\ wang0822@umn.edu
}

\author{
Zhifeng Tao \\ Mitsubishi Electric Research \\ Laboratories (MERL) \\ 201 Broadway \\ Cambridge, MA 02139, USA \\ tao@merl.com
}

\author{
Andreas F. Molisch \\ Ming Hsieh Department of \\ Electrical Engineering \\ Univ. of Southern California \\ Los Angeles, CA 90089, USA \\ molisch@usc.edu
}

\author{
Philip V. Orlik \\ Mitsubishi Electric Research \\ Laboratories (MERL) \\ 201 Broadway \\ Cambridge, MA 02139, USA \\ porlik@merl.com
}

\author{
Jinyun Zhang \\ Mitsubishi Electric Research \\ Laboratories (MERL) \\ 201 Broadway \\ Cambridge, MA 02139, USA \\ jzhang@merl.com
}

\begin{abstract}
The IEEE 802.16/WiMAX standard has fully embraced multiantenna technology and can, thus, deliver robust performance and high transmission rates. Nevertheless, due to its inherent cost concerns, a WiMAX mobile station (MS) should preferably contain fewer radio frequency (RF) chains than antenna elements. Thus, antenna selection, wherein a subset of antennas is dynamically selected to connect to the limited number of RF chains for transceiving, is a highly appealing performance enhancement technique for multiantenna WiMAX terminals. In this paper, a novel protocol for antenna selection in space division multiple access (SDMA) transmission is developed for the next-generation IEEE 802.16 mobile stations. Both locally and globally optimal selection rules are considered at the base station (BS). The proposed protocol can readily accommodate various channel situations (e.g., reciprocal and non-reciprocal channels). As demonstrated by analysis and simulation, the proposed protocol delivers considerable performance improvement over conventional IEEE 802.16 terminals that lack antenna selection capability. Moreover, the proposed protocol leverages the existing signaling method defined in IEEE 802.16, thereby incurring a negligible signaling overhead and requiring only minimal modifications of the standard.
\end{abstract}

\section{Categories and Subject Descriptors}

C.2.1 [Network Architecture and Design]: Wireless Communication

Permission to make digital or hard copies of all or part of this work for personal or classroom use is granted without fee provided that copies are not made or distributed for profit or commercial advantage and that copies bear this notice and the full citation on the first page. To copy otherwise, to republish, to post on servers or to redistribute to lists, requires prior specific permission and/or a fee.

IWCMC '09, June 21 - 24, 2009, Leipzig, Germany

Copyright 2009 ACM 978-1-60558-569-7/09/06 ...\$5.00.

\section{General Terms}

Algorithms, performance, standardization

\section{Keywords}

Antenna selection, spatial division multiple access (SDMA), protocol design, WiMAX

\section{INTRODUCTION}

In order to achieve the capacity and quality of service (QoS) demanded by high-speed multimedia services, both the original WiMAX IEEE 802.16 standard and its subsequently ratified amendments make use of a large number of advanced technologies. The current IEEE 802.16 standard is based on MIMO-OFDMA (where MIMO stands for "multiple input multiple output" and OFDMA for "orthogonal frequency division multiple access") [12]. A further technology enhancement foreseen in the standard is the use of space division multiple access (SDMA), a multi-user MIMO technology that can enable a base station (BS) to transmit (or receive) signal to (or from) multiple mobile stations (MSs) concurrently using the same channel resource, namely the same time slot and frequency subchannels.

At an MS with multiple antennas, the number of radio frequency $(\mathrm{RF})$ chains $\left(N_{R F}\right)$ is often less than the number of antennas $\left(N_{R}\right)$, i.e., $N_{R F} \leq N_{R}$, due to the high cost of RF chains and relatively low cost of antennas. In a wireless propagation channel, it is known that each antenna provides a distinct signal path that experiences different fading. Therefore, it is important to perform antenna selection, which selectively and dynamically connects a subset of cardinality $N_{R F}$ of the available antennas to the RF chains. The criterion for selecting the antennas is the improvement of system performance in terms of bit error rate (BER), signal to noise ratio (SNR) or throughput. Theoretical aspects of antenna selection have already been studied extensively in the literature $[7,10]$ and references therein. Antenna selection has also recently been incorporated as a key feature in the high speed IEEE 802.11n wireless local area network (WLAN) standard [2], and accepted as a primary working 
assumption in the 3GPP LTE standard [5].

In the current IEEE 802.16 networks, however, antenna selection is supported only at the BS side, essentially as a coarse but robust precoding technique [6]. Indeed, the WiMAX standard has not exploited antenna selection at MSs, where cost and complexity concerns are paramount and antenna selection is needed the most. Recently, Nie et al. [8] proposed an antenna selection protocol for IEEE 802.16 MSs for a single-user MIMO system. However, to the best knowledge of the authors, MS antenna selection for SDMA transmission has never been discussed in the context of WiMAX networks.

In this paper we propose a protocol called $M A S S$ for MS Antenna Selection for SDMA transmissions in order to achieve local or global optimality in WiMAX networks. One salient feature of this proposed protocol is that it can support all the major permutations (i.e., assignment of transmit symbols to OFDM-subcarriers) defined in IEEE 802.16, and adaptively operates under both reciprocal and non-reciprocal channel conditions for downlink and uplink. Since the MASS protocol substantially reuses the channel measurement and reporting, media access (MAC) messages, and physical (PHY) support that have already been provided by the current IEEE 802.16 standard, it only incurs diminutive additional complexity, thereby speeding up its acceptance and implementation.

We will use the following notation: Upper and lower case bold symbols denote matrices and column vectors, respectively. $(\cdot)^{T}$ stands for transpose, $(\cdot)^{\mathcal{H}}$ Hermitian transpose and $\|\cdot\|_{F}$ Frobenius norm. $\mathcal{C N}\left(0, \sigma^{2}\right)$ represents the circularly symmetric complex Gaussian distribution with zero mean and variance $\sigma^{2} ; \bar{\gamma}=E\{\gamma\}$ is the mean of a random variable $\gamma$.

\section{PROTOCOL FOR MS ANTENNA SELEC- TION IN SDMA (MASS)}

\subsection{Antenna Switching}

In order to determine the channel state between all the possible transmit antenna subsets and the receive antenna subsets, all the available antenna subsets need to sound the channel, i.e., send or receive pilot symbols. This entails switching between different antenna subsets, which can occur during the cyclic prefix (CP) interval of an OFDM symbol [9].

Antenna selection at MS can be classified as transmit antenna selection (TAS), which occurs in the uplink (UL), and receive antenna selection (RAS), which occurs in the downlink (DL). The algorithms selecting the antenna elements subset can be categorized as local selection (LS) and global selection (GS). As the name suggests, LS only considers information available at the MS on which a subset of antennas need to be selected, thereby rendering a locally optimal performance. In contrast, GS takes channel information for all the MSs involved in the same SDMA transmission into account, and thus can yield the optimal subset of antennas. Note that it is the BS that determines whether locally optimal selection or globally optimal selection should be used. We will first describe the proposed algorithms for LS and GS, and then introduce the adaptive $M A S S$ protocol to provide a unified framework that can cover all pertinent scenarios.

\subsection{Local Selection Algorithm}

For LS at a designated MS, the antenna selection (AS) is based on the channel state information (CSI) between this MS and the BS only. Note that MSs other than the designated MS do not have to switch antennas in this process, as the antenna selection on this MS does not affect the selected antennas on other MSs. Since the proposed algorithm is identical for all MSs, it is sufficient to describe the local AS for just one MS, say $\mathrm{MS}_{k}$. The AS can either be scheduled to occur on a periodic basis (which can be related to estimates of the channel coherence time) or performed "on demand". In the latter case, AS is usually initiated by the receiver.

\section{Table 1:}

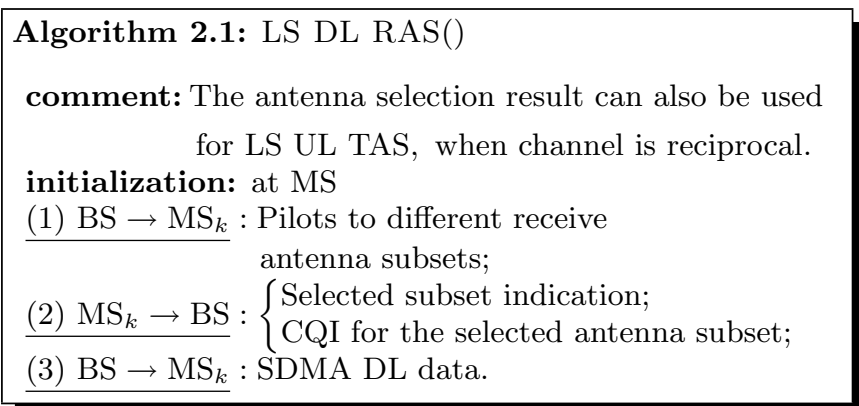

For LS DL RAS, the algorithm is depicted in Algorithm 2.1 (Table 1). Once the designated MS $\left(M S_{k}\right)$, which is the receiver in the downlink, decides to initiate antenna selection, it uses different subsets of antennas to receive different OFDMA symbols that contain pilot subcarriers, and estimates the CSI of the channel associated with each different subset of antennas. The MS then can select the antenna subset that will give it the best channel quality. The full CSI or just channel quality information (CQI) should be fed back from the MS to BS for precoding before SDMA transmissions. The MS can also inform the BS of the the identity of the selected antenna subset, so that BS can track the channel variation or user mobility by comparing the CQI feedback with the stored CQI of the corresponding antenna subset.

If the channel is reciprocal, the same antenna subset selected for reception on the downlink can then be used for transmission on the uplink. Otherwise, the BS transmits an MS antenna selection for SDMA uplink information element (MASS_UL_IE) to the MS to initiate the UL TAS process, as depicted in Algorithm 2.2 (Table 2). After being notified by the BS, the MS uses the current antenna subset to transmit pilot signals in the first available pilot symbol, and then sequentially uses disjoint antenna subsets to transmit subsequent pilot signals. Thus, the BS can estimate the channel response associated with each different antenna subset used by the MS in the uplink, and determine the optimal transmit antenna subset that the MS should use. The BS then sends a 2-byte long MASS_UL_IE to notify the MS of this optimal subset. The MS should follow the instruction and switch to the selected antenna subset to transmit subsequent packets in the uplink subframe.

\subsection{Global Selection Algorithm}

When multiple MSs participate in SDMA communications by using the same channel resource to transmit in the uplink or receive in the downlink concurrently, globally opti- 
mal antenna selection can be used. In contrast to LS, the GS for each designated MS depends on not only the channel between BS and this particular MS, but also the channel between BS and all other MSs engaging in the SDMA transmissions.

LS selects the antennas that will yield the highest noiselimited capacity. However, if applying the same criteria for SDMA communications, an antenna subset that is not globally optimal may be chosen. Indeed, what is different for GS is the fact that the interference that one MS creates to another is taken into account. It might thus be preferable to pick certain antenna elements that do not maximize the noise-limited capacity for a given MS, but create less interference to other MSs.

Table 2:

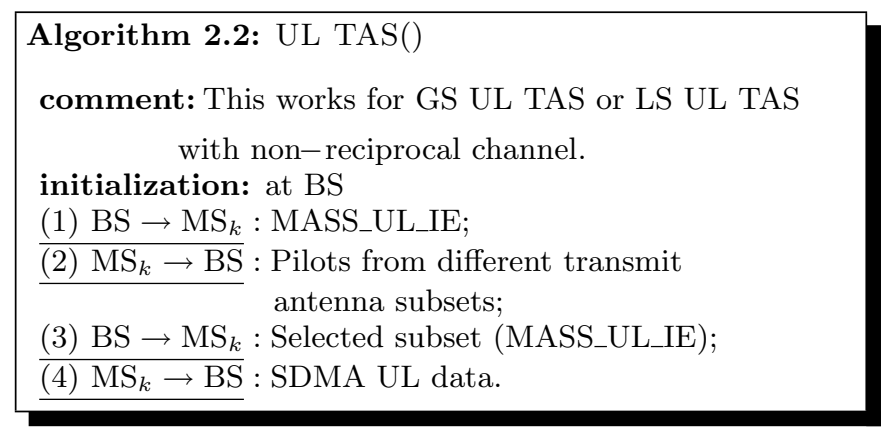

When using GS in an SDMA communications, the selection decision is made at the BS, which therefore needs to have access to the measured CSI of all the channels involved. The uplink TAS is normally triggered by the BS using MASS_UL_IE. In response, the designated MS transmits the OFDMA symbol with pilots using different antenna subsets. After the BS collects the CSI from all MSs, globally optimal criteria can be applied to select antenna subset for each MS. The BS indicates the selection in the MASS_UL_IE to the MS, which will switch to the selected antenna subset for subsequent uplink data transmissions. Notice that this procedure is identical to the LS UL TAS depicted in Algorithm 2.2, except that the GS criterion is used at the BS.

For the downlink, however, both MS and BS can initiate the RAS. Each MS measures the CSI between the BS and different antenna subsets, and feeds back the CSI to the BS. Again, the decision of which antenna subset to use is made by the BS, after globally considering the CSI from all MSs.

Table 3:

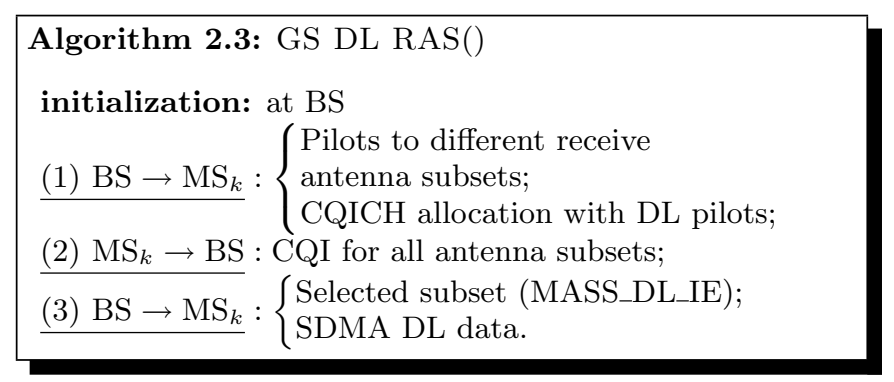

Algorithm 2.3 (Table 3) describes the GS DL RAS procedure initiated by the BS. In order to let MS report the channel quality it has measured, the BS needs to allocate to the MS an uplink channel quality information (CQI) channel $(\mathrm{CQICH})$ by using information elements defined in current IEEE 802.16 (e.g., CQICH_Allocation_IE, etc.).

Based upon the CQI report received from the MS, the BS can select the subset of antennas for MS to use in downlink for reception. The BS indicates the selected antenna subset in MASS_DL_IE to the MS for its subsequent SDMA downlink data reception.

The MASS_DL_IE is an extended DL-MAP IE to support antenna selection signaling, and has a structure that is similar to MASS_UL_IE. Note that the format of both MASS_UL_IE and MASS_DL_IE complies with that of a general IEEE802.16 information element. Due to space limitation, however, the detailed format of these two information elements is omitted here.

\section{Table 4:}

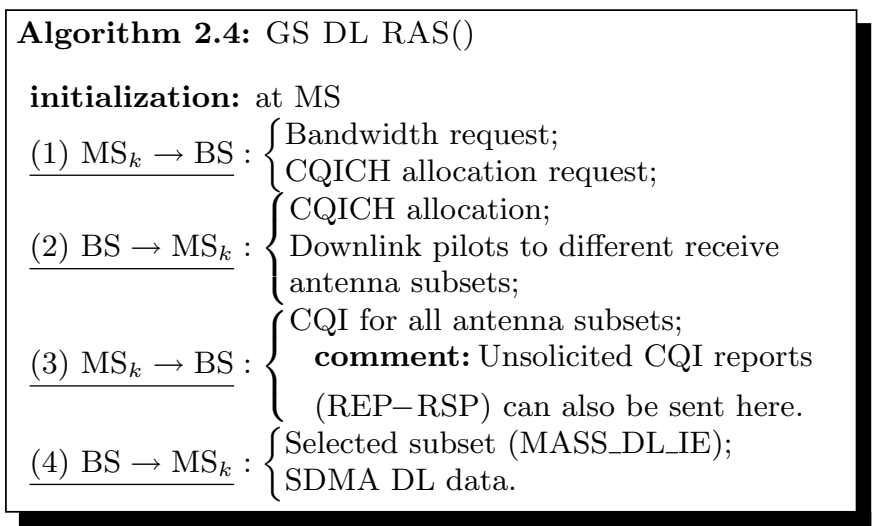

Algorithm 2.4 (Table 4) depicts the procedure for MSinitiated GS DL RAS. The DL RAS procedure sometimes should be initiated by MS, for example, if the latest CQI report sent to the $\mathrm{BS}$ is no longer up-to-date while the next periodic CQI feedback opportunity is still far away.

In this case, $\mathrm{MS}_{k}$ can initiate the $\mathrm{DL} \mathrm{RAS}$, and request bandwidth from the BS for channel quality reporting by sending a CQICH allocation request. In order to send a $\mathrm{CQICH}$ allocation request, however, the MS has to first acquire proper resources for uplink transmission. An MS can use various bandwidth request (BR) schemes and contentionresolution protocol defined in the current IEEE 802.16 standard to acquire such uplink resource. Once an uplink transmission opportunity is secured, the MS can use it to request a $\mathrm{CQICH}$ allocation. The $\mathrm{BS}$ allocates the resource for CQICH reporting to the MS, and transmits the pilot signals along with downlink data. The MS receives the pilot signals using different subset of antennas, and estimates the downlink channel associated with each antenna subset based upon the pilots. The MS informs the BS of the CQI for the channel associated with each antenna subset in the allocated CQICH channel.

Sometimes the last CQI report of the MS is no longer appropriate for the remaining duration until the next periodic CQI feedback. In this case, the MS can transmit the unsolicited CQI report to the BS through a report response (REP-RSP) message. The MS has to ensure in advance that there is sufficient bandwidth for the transmission of a REP-RSP message in the uplink via such methods as contention-based bandwidth request. The BS notifies the MSs by transmitting MASS_DL_IE to each MS before an 
SDMA downlink transmissions.

\subsection{Adaptive MASS Protocol}

We now define the $M A S S$ protocol which is consistent with the current IEEE 802.16 specifications and efficiently activates antenna selection operations whenever necessary. The proposed protocol can support all permutations defined in the standard and accommodate both LS and GS criteria under both reciprocal and non-reciprocal channel conditions.

As illustrated in Fig. 1, when an MS enters an IEEE 802.16 network, it transmits a subscriber station basic capability request (SBC-REQ) message, which is defined by the current standard to indicate the functionalities that the transmitting MS can support. This message has to communicate to the BS the number of antenna elements and RF chains that the MS contains, and whether the MS supports antenna selection functionality or not. Then, the BS sends back a subscriber station basic capability response (SBCRSP) message as an acknowledgment. By exchanging SBCREQ and SBC-RSP messages, BS and MS learn about basic physical parameters of each other.

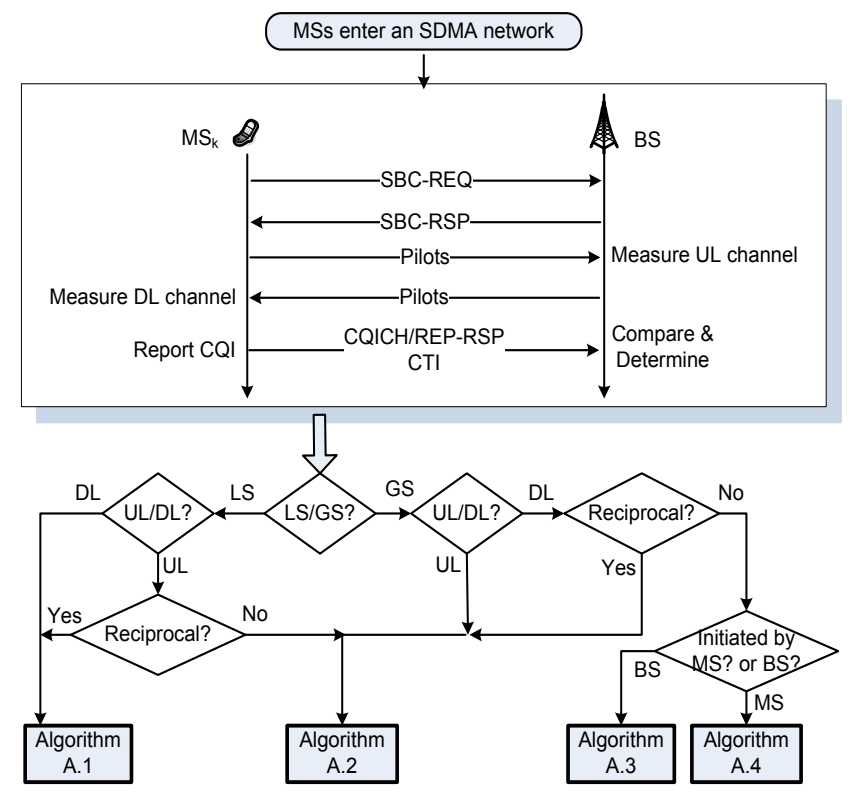

Figure 1: Adaptive antenna selection protocol for SDMA transmission in IEEE 802.16 system.

In the IEEE 802.16 time-division-duplex (TDD) system, the uplink and downlink transmissions share the same frequency spectrum. Accordingly, the channel state in an IEEE 802.16 network is measured in the downlink and uplink within a downlink subframe and uplink subframe by MS and BS, respectively. The channel estimation is conducted by measuring pilot signals, which are carried within pilot symbols and subcarriers. When the MS receives pilot signals from the $\mathrm{BS}$, it feeds back the measurement results to the BS through the CQICH or by means of a channel measurement report response (REP-RSP) message, as defined in IEEE802.16 standard. The BS, upon receiving channel measurements from an MS, compares the downlink and uplink channel estimates and then determines whether the DL and uplink channels are reciprocal or not.

In addition to CQI, each MS should also report to the
BS the coherence time index (CTI), which indicates the coherence time information for the channels between the MS and BS. This allows the BS to determine whether LS or GS should be adopted. For example, if one user's channel coherence time is much shorter than that of the other users', it implies that this particular user is probably moving at a fairly high speed. Thus, it is more appropriate to apply LS for this user, while using GS for other users with similar channel coherence time.

For LS, the DL protocol follows Algorithm 2.1 described in Section 2.2. In the uplink, it depends on whether the downlink and uplink channels are reciprocal. If channel reciprocity holds, an MS should transmit in the uplink with the antennas selected per Algorithm 2.1. Otherwise, the MS should perform AS according to Algorithm 2.2.

For GS in uplink, Algorithm 2.2 still works with GS criteria. In the downlink, if the channels are reciprocal, the MS can use the same antenna subset chosen for UL TAS transmissions following Algorithm 2.2. But if the channels are not reciprocal, two cases may happen depending on whether the AS is initiated by MS or BS, which should follow Algorithms 2.3 and 2.4 , respectively.

In summary, with different algorithms described in sections 2.2 and 2.3, this adaptive MASS protocol can handle all the scenarios including LS and GS, DL and UL, in both reciprocal and non-reciprocal channel states.

\section{ANTENNA SELECTION CRITERIA AND PERFORMANCE}

This section evaluates the performance of the adaptive MASS protocol with various antenna selection criteria. Since SDMA enables a BS to transmit to (or receive from) multiple MSs concurrently using the same channel resource, it can substantially improve network capacity. With antenna selection implemented in SDMA, further improvement of the capacity is expected.

\subsection{System Model}

We consider a cellular SDMA system with one BS (with $N_{T}$ antennas and $N_{T} \mathrm{RF}$ chains) and $K$ MSs/users (each with $N_{R}$ antennas and $N_{R F} \mathrm{RF}$ chains). The BS transmits $L_{k}$ data streams to MS $k$. Different links are assumed to be independent and undergo frequency-flat Rayleigh fading. ${ }^{1}$ Therefore, the baseband matrix representation of the channel from BS to MS $k, \mathbf{H}_{k}$, has complex Gaussian elements $h_{j i}$ from transmit antenna $i$ to receive antenna $j$, $i=1, \ldots, N_{T}, j=1, \ldots, N_{R}$. The transmit vector corresponding to MS $k$ is linearly precoded by the $N_{T} \times L_{k}$ matrix $\mathbf{T}_{k}$ as $\mathbf{x}_{k}(m)=\mathbf{T}_{k} \mathbf{s}_{k}(m)$, where $\mathbf{s}_{k}(m)$ denotes the zero-mean data vector, of size $L_{k} \times 1$ at time $m$. Our protocol from Section 2 enables the BS to have complete CSI for all the BS-MS channels; note that this assumption had been used in previous theoretical papers $[3,11,13]$ though those papers did not specify protocols for obtaining this CSI. We also assume a block-fading channel model with a sufficiently large coherence time so that the channel fading remains the same over the duration in which $\mathbf{T}_{k}$

\footnotetext{
${ }^{1}$ The difference between the performance of local selection and global selection is not pronounced under this assumption. Note that the performance of a real system will depend on not only channel configurations, but also many other factors such as type of permutations used, code rate, etc.
} 
is used. Given current CSI, in order to maximize the peruser transmission information rate, a Gaussian code book is used for the transmit data vectors, with normalized power such that $E\left[\mathbf{s}_{k}(m) \mathbf{s}_{k}(m)^{\mathcal{H}}\right]=\mathbf{I}_{L_{k}}$. Furthermore, the code books for different users are independent of each other, i.e., $E\left[\mathbf{s}_{k}(m) \mathbf{s}_{l}(m)^{\mathcal{H}}\right]=\mathbf{0}$, for $k \neq l$. These assumptions make the simulations feasible, though we note that the actual IEEE 802.16 standard only approximates the Gaussian codebook with a finite set of modulation/coding techniques.

The equivalent discrete-time received signal corresponding to symbol $m$ of user $k$ after matched filtering and sampling can be expressed as

$$
\mathbf{y}_{k}(m)=\mathbf{H}_{k} \mathbf{T}_{k} \mathbf{s}_{k}(m)+\sum_{j=1, j \neq k}^{K} \mathbf{H}_{k} \mathbf{T}_{j} \mathbf{s}_{j}(m)+\mathbf{n}_{k}(m)
$$

where $\mathbf{n}_{k}(m)$ is the additive white Gaussian noise vector, $k=1, \ldots, K$.

\subsection{Antenna Selection Criteria}

Either LS or GS criteria can be used for antenna selection in SDMA. For LS, each MS selects antenna(s) without knowledge of the channels between BS and other MSs. Consequently, one reasonable metric for both downlink and uplink is to maximize the capacity for each MS. If we further assume $N_{0}=1$ in the discussion hereafter, this would imply finding the best antenna subsets for MS $k$ that maximize

$$
C_{k}=\log \left|\mathbf{I}+\mathbf{H}_{k} \mathbf{H}_{k}^{\mathcal{H}}\right| \text {. }
$$

Notice that $\mathbf{T}_{k}$ can not be used for LS in (2), as $\mathbf{T}_{k}$ is actually a function of $\mathbf{H}=\left[\mathbf{H}_{1}, \ldots, \mathbf{H}_{K}\right]$. Also note that we do not assume the column of $\mathbf{T}_{k}$ to be unitary, but instead incorporate the power control in the precoding matrix.

For GS, however, the BS should make use of the CSI from all users, as well as the precoding matrix derived from the CSI. The sum rate of the SDMA system is used as the metric for GS in the uplink, i.e., the selected antenna(s) on the users should maximize

$$
C_{\text {sum }}=\log \left|\mathbf{I}+\mathbf{H T T}^{\mathcal{H}} \mathbf{H}^{\mathcal{H}}\right|
$$

where $\mathbf{T}=\left[\mathbf{T}_{1}, \ldots, \mathbf{T}_{K}\right]$. GS can also be performed by maximizing the sum of signal to interference plus noise ratios (SINRs):

$$
\mathrm{SINR}_{\text {sum }}=\sum_{k=1}^{K} \frac{\left\|\mathbf{H}_{k} \mathbf{T}_{k}\right\|_{F}^{2}}{\sum_{j=1, j \neq k}^{K}\left\|\mathbf{H}_{k} \mathbf{T}_{j}\right\|_{F}^{2}+N_{R}} .
$$

Or, to take fairness into consideration, BS can globally select antennas by maximizing the minimum user rate, i.e.,

$$
C_{\min }=\min _{k=1, \ldots, K} \log \left|\mathbf{I}+\mathbf{H}_{k} \mathbf{T}_{k} \mathbf{T}_{k}^{\mathcal{H}} \mathbf{H}_{k}^{\mathcal{H}}\right| .
$$

Furthermore, to guarantee a QoS requirement of e.g., minimum rate for each user, antennas should be selected to maximize $C_{\text {sum }}$ in (3) while given the constraint of $C_{\min } \geq C_{0}$, for some $C_{0}>0$.

In order to find the optimum antennas, we can use an exhaustive search over all possible antenna subset combinations over all MSs, where for each subset combination we optimize the cost function according to one of the metrics in (2)-(5). With $K$ users in an SDMA system, the complexity of the GS algorithm grows exponentially with $K$. To be specific, the complexity is on the order of $\Theta\left(\prod_{k=1}^{K} \alpha_{k}\right)$, where $\alpha_{k}=\left(\begin{array}{c}N_{R} \\ N_{R F}\end{array}\right)$ denotes the number of antenna subsets for user

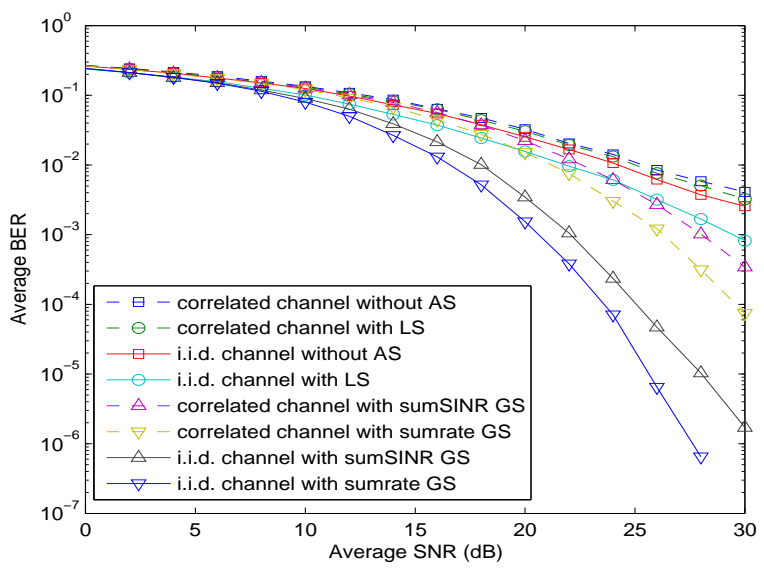

Figure 2: Average BER comparison for SDMA system with various selection criteria $\left(N_{T}=4, N_{R F}=2\right.$, $\left.N_{R}=4, K=2\right)$.

$k$. In this case, the dimensionality of the problem (number of possible subsets) explodes with the number of users. In practice, however, since SDMA in an OFDMA system usually does not assign more than 3 or 4 users to the same resource, this exhaustive search remains a viable solution approach. As a result, there is no analytical expression for the optimal solution of the antenna selection, which depends on the channel properties and antenna selection criteria.

\section{SIMULATION RESULTS}

Using the channel model described above, simulations in this subsection compare BER and sum rate of antenna selection schemes in SDMA for practical SNR values [4]. Unless otherwise stated, we will consider the SDMA system with $N_{T}=4, N_{R F}=2, N_{R}=4, K=2, L_{1}=L_{2}=1$, and the default setup is with sum-rate GS and uncoded QPSK modulation.

Fig. 2 shows the BER for AS with various selection criteria in SDMA systems. It is evident that in both correlated and uncorrelated channels, LS slightly outperforms the case without AS, while GS gives a tremendous improvement compared to LS. GS has a much better diversity gain compared to LS and the case without AS, meaning a steeper slope of the BER vs. SNR curve. Combining the results with the complexity discussion in Section 3.2, we conclude that when there are just 2 to 4 users in SDMA systems, GS should be used for the MASS protocol. For GS, moreover, sum-rate (3) performs better than sum-SINR (4) and is actually the best among all selection criteria. Thus, we use sum-rate GS as our default selection criterion from now on. Fig. 2 also shows that for the same scheme, channel correlation may have significant negative impact on the performance of antenna selection.

Performance comparisons for SDMA systems with various numbers of users are given in Fig. 3, based on sum rate GS. Here we set $N_{T}=4, N_{R F}=1$ and $N_{R}=2$. As expected in Fig. 3(a), improved average BER is achieved as the number of users $K$ decreases. Note that, when $K=1$, the system is reduced to a co-located MIMO system. It seems that the gaps between the BER curves become smaller as the number of users increases, implying that more users are preferred in the MASS protocol for sum rate GS whenever 


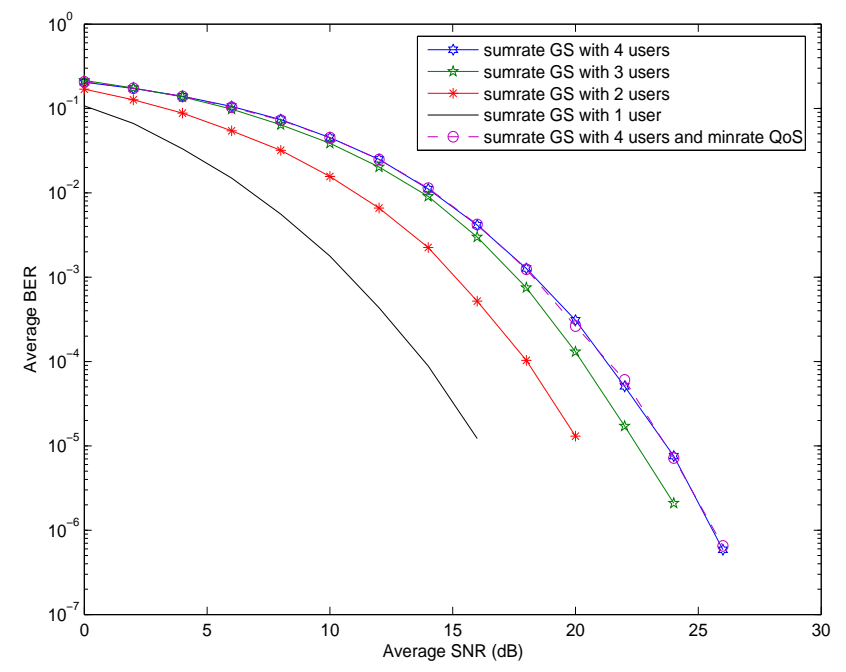

(a) Average BER

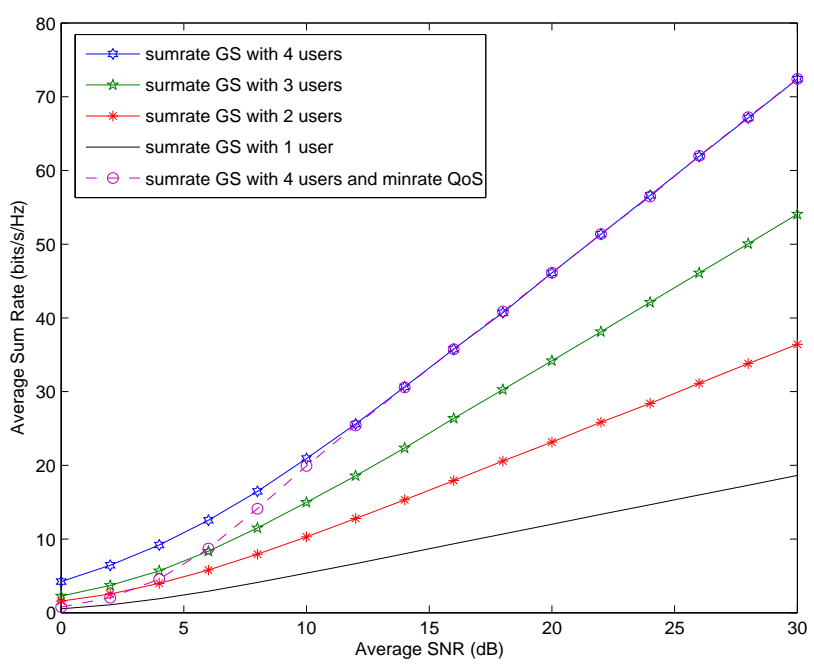

(b) Average Sum Rate

Figure 3: SDMA system with multiple users (sum-rate GS, $N_{T}=4, N_{R F}=1, N_{R}=2$ ).

the complexity can be afforded in practice. This can be further confirmed by Fig. 3(b), which shows that sum rate also grows significantly as $K$ increases. To address the QoS issues, Fig. 3 also depicts the sum rate GS for 4 users with a minimum rate per user $C_{\min } \geq \log (1+\mathrm{SNR})$. The minimum rate constraint does not degrade the average BER as shown in Fig. 3(a), and also retains the sum rate at high SNRs, though it does degrade the sum rate in the low SNR regime, see Fig. 3(b). This implies that at high SNR regime, the antenna subsets maximizing sum rate satisfy at the same time the QoS requirement of minimum rate per user.

\section{CONCLUSIONS}

This paper proposes the use of antenna selection as an efficient and cost-effective solution for enhancing SDMA system performance for next generation IEEE 802.16 mobile terminals [1]. For each designated MS, a locally or globally optimal subset of antennas is selected based on the CSI. A

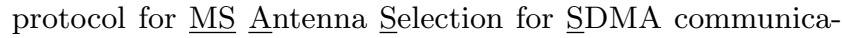
tions (MASS) is proposed to accommodate both reciprocal and non-reciprocal channel conditions. As verified by simulations, MASS protocol substantially improves the BER and sum rate performance, and incurs a negligible signaling and implementation complexity overhead.

\section{REFERENCES}

[1] IEEE 802.16 Task Group m (TGm). Official IEEE 802.16m Website: http: // ieee802. org/16/tgm/.

[2] M. Collados and A. Gorokhov. Antenna selection for MIMO-OFDM WLAN systems. In 15th IEEE International Symposium on Personal, Indoor and Mobile Radio Communications, volume 3, pages 1802-1806, Sept. 2004.

[3] A. Goldsmith, S. A. Jafar, N. Jindal, and S. Vishwanath. Capacity limits of MIMO channels. IEEE Journal on Selected Areas in Communications, 21(5):684-702, June 2003.

[4] IEEE $802.16 \mathrm{~m}$. Draft ieee $802.16 \mathrm{~m}$ evaluation methodology. IEEE Methodology, Oct. 2007.
[5] Institute for Infocomm Research (I2R), Mitsubishi Electric, and NTT DoCoMo. Transmit Antenna Selection Techniques for Uplink E-UTRA. In 3GPP RAN1, R1-051398, Seoul, Korea, November 2005.

[6] E. Kurniawan, A. S. Madhukumar, and F. Chin. Exploiting antenna selection gain in IEEE 802.16 broadband wireless access systems. In 10th IEEE International Conference on Communication systems, pages 1-5, Singapore, Oct. 2006.

[7] A. F. Molisch and M. Z. Win. MIMO systems with antenna selection. IEEE Microwave Magazine, 5(1):46-56, Mar. 2004.

[8] C. Nie, Z. Tao, N. B. Mehta, A. F. Molisch, J. Zhang, T. Kuze, and S. Panwar. Antenna selection for next generation IEEE 802.16 mobile stations. In International Conference on Communications, Beijing, China, May 2008, accepted.

[9] A. Partridge, P. Acsadi, G. Omictin, J. Robinson, and H. J. Henderson. Switched charge voltage driver and method for applying voltage to tunable dielectric devices. U.S. Patent No. 6,535,0\%6, Mar. 2003.

[10] S. Sanayei and A. Nosratinia. Antenna selection in MIMO systems. IEEE Communications Magazine, 42(10):68-73, Oct. 2004.

[11] S. Shamai and B. M. Zaidel. Enhancing the cellular downlink capacity via co-processing at the transmitting end. In Vehicular Technology Conference, 2001. VTC 2001 Spring. IEEE VTS 53rd, volume 3, pages 1745-1749, Rhodes, May 2001.

[12] G. L. Stuber, J. Barry, S. McLaughlin, Y. G. Li, M. A. Ingram, and T. Pratt. Broadband MIMO-OFDM wireless communications. Proceedings of the IEEE, 92:271 - 294, Feb. 2004.

[13] H. Zhang and H. Dai. Cochannel interference mitigation and cooperative processing in downlink multicell multiuser MIMO networks. EURASIP Journal on Wireless Communications and Networking, 2:222-235, 2004 . 